PROCEEDINGS OF THE

AMERICAN MATHEMATICAL SOCIETY

Volume 138, Number 5, May 2010, Pages 1785-1791

S 0002-9939(09)10211-3

Article electronically published on December 3, 2009

\title{
NONEXISTENCE OF HORIZONTAL SOBOLEV SURFACES IN THE HEISENBERG GROUP
}

\author{
VALENTINO MAGNANI
}

(Communicated by Mario Bonk)

\begin{abstract}
Involutivity is a well known necessary condition for integrability of smooth tangent distributions. We show that this condition is still necessary for integrability with Sobolev surfaces. We specialize our study to the left invariant horizontal distribution of the first Heisenberg group $\mathbb{H}^{1}$. Here we answer a question raised in a paper by Z.M. Balogh, R. Hoefer-Isenegger, and J.T. Tyson.
\end{abstract}

The Heisenberg group $\mathbb{H}^{1}$ can be represented as $\mathbb{R}^{3}$, equipped with the couple of left invariant vector fields

$$
X_{1}(x)=\partial_{x_{1}}-x_{2} \partial_{x_{3}}, \quad X_{2}(x)=\partial_{x_{2}}+x_{1} \partial_{x_{3}}
$$

with respect to the group operation $x y=x+y+\left(0,0, x_{1} y_{2}-x_{2} y_{1}\right)$, for every $x, y \in \mathbb{R}^{3}$. In the sequel, we will use the standard Euclidean norm $|\cdot|$ on $\mathbb{H}^{1}$, especially when we consider rectifiable sets, in the Federer sense. We denote by $\mathcal{H}_{|\cdot|}^{\alpha}$ the $\alpha$-dimensional Hausdorff measure with respect to $|\cdot|$. Sobolev mappings with values in $\mathbb{H}^{1}$ are thought of as having values in $\mathbb{R}^{3}$. The measures $\mathcal{S}^{\alpha}$ and $\mathcal{H}^{\alpha}$ are assumed to be constructed with respect to a fixed left invariant homogeneous distance of the Heisenberg group.

Definition 1. We say that a countably $\mathcal{H}_{|\cdot|}^{2}$-rectifiable set $S$ of $\mathbb{H}^{1}$ is a Sobolev surface if it can be written, up to $\mathcal{H}_{|\cdot|}^{2}$-negligible sets, as the countable union of graphs of precisely represented Sobolev functions of class $W_{l o c}^{1,1}$ and defined on open sets of $\mathbb{R}^{2}$.

Remark 1. In view of recent results by J. Malý, D. Swanson and W. P. Ziemer, graphs of precisely represented functions in our assumptions are countably $\mathcal{H}_{|\cdot|^{-}}^{2}$ rectifiable; see [10. Then the hypothesis of rectifiability in Definition 1 could be removed.

The distribution of admissible directions in the Heisenberg group is given by the following horizontal subspaces:

$$
H_{x} \mathbb{H}^{1}=\left\{\lambda_{1} X_{1}(x)+\lambda_{2} X_{2}(x) \mid \lambda_{j} \in \mathbb{R}\right\} \quad \text { for every } x \in \mathbb{H}^{1} .
$$

The collection of all horizontal subspaces $H_{y} \mathbb{H}^{1}, y \in \mathbb{H}^{1}$, seen as a subbundle of $T \mathbb{H}^{1}$, is the so-called horizontal subbundle and it is denoted by $H \mathbb{H}^{1}$.

Received by the editors March 19, 2009, and, in revised form, September 11, 2009.

2000 Mathematics Subject Classification. Primary 26B99; Secondary 28A78, 53C17.

(C)2009 American Mathematical Society 
Definition 2. A countably $\mathcal{H}_{|\cdot|}^{2}$-rectifiable set $S$ in $\mathbb{H}^{1}$ is horizontal if for $\mathcal{H}_{|\cdot|}^{2}$-a.e. $x \in S$, we have $\operatorname{Tan}(S, x) \subset H_{y} \mathbb{H}^{1}$. We also say that $S$ is $\mathcal{H}_{|\cdot|}^{2}$-a.e. tangent to $H \mathbb{H}^{1}$. A Sobolev surface is horizontal if it is $\mathcal{H}_{|\cdot|}^{2}$-a.e. tangent to $H \mathbb{H}^{1}$.

Remark 2. Then the nonexistence of horizontal smooth 2-dimensional submanifolds in $\mathbb{H}^{1}$ is a simple consequence of the fact that the horizontal distribution given by horizontal subspaces $H_{y} \mathbb{H}^{1}$ is noninvolutive. In fact, $\left[X_{1}, X_{2}\right]=2 \partial_{y_{3}}$ and this vector field clearly is not a linear combination of $X_{1}$ and $X_{2}$.

Lemma 1. Let $f \in W_{\text {loc }}^{1,1}\left(\Omega, \mathbb{H}^{1}\right)$ be a graph parametrization of a Sobolev surface. Then there exists a set of positive measure in $\Omega$ such that the system

$$
\left\{\begin{array}{l}
f_{x_{1}}^{3}=f^{1} f_{x_{1}}^{2}-f^{2} f_{x_{1}}^{1} \\
f_{x_{2}}^{3}=f^{1} f_{x_{2}}^{2}-f^{2} f_{x_{2}}^{1}
\end{array}\right.
$$

fails to hold in all points of this set.

Proof. Recall that $\Omega$ is an open subset of $\mathbb{R}^{2}$. We can rewrite the system (1) in terms of differential forms as the a.e. pointwise validity of

$$
d f^{3}=f^{1} d f^{2}-f^{2} d f^{1} .
$$

Since $f$ parametrizes a graph, it can be represented in three possible ways, where it always happens that either $f^{1}$ or $f^{2}$ is a coordinate function. Thus, one of these components clearly is in $W_{l o c}^{1,1}(\Omega)$ and the remaining one is smooth. As a consequence, both $f^{1} d f^{2}$ and $f^{2} d f^{1}$ can be weakly differentiated and the weak exterior differential satisfies the formula

$$
d\left(f^{1} d f^{2}-f^{2} d f^{1}\right)=2 d f^{1} \wedge d f^{2} .
$$

Clearly, $d\left(d f^{3}\right)=0$ in the distributional sense; hence

$$
\int_{\Omega} *\left(d f^{1} \wedge d f^{2}\right) \phi d \mathcal{L}^{2}=0
$$

for $\phi \in C_{c}^{\infty}(\Omega)$, where $*\left(d f^{1} \wedge d f^{2}\right)=\operatorname{det}\left(f_{x_{j}}^{i}\right)$. We have proved that $\nabla f^{1}(x)$ and $\nabla f^{2}(x)$ are not linearly independent for a.e. $x \in \Omega$. Due to (1), it follows that the rank of $\nabla f(x)$ is less than or equal to one for a.e. $x \in \Omega$. This conflicts with the fact that $f$ parametrizes a graph.

Remark 3. In the previous proof we have used the notion of weak exterior differential of a locally summable $k$-form $\alpha$ on an open set $\Omega$ of $\mathbb{R}^{n}$. Recall that the locally summable $(k+1)$-form $\beta$ is the weak exterior differential of $\alpha$ if for every smooth compactly supported $(n-k-1)$-form $\phi$, we have

$$
\int_{\Omega}\langle\alpha, * d \phi\rangle d \mathcal{L}^{n}=(-1)^{k+1} \int_{\Omega}\langle\beta, * \phi\rangle d \mathcal{L}^{n} .
$$

Here $*$ denotes the Hodge operator with respect to the volume form $d x_{1} \wedge \cdots \wedge d x_{n}$. Notice also that $\beta$ is uniquely defined. The validity of the formulae $d\left(f^{1} d f^{2}\right)=$ $d f^{1} \wedge d f^{2}$ and $d\left(f^{2} d f^{1}\right)=d f^{2} \wedge d f^{1}$ used in the previous proof can be obtained by standard smooth approximation arguments.

Remark 4. One can check that the pointwise validity of (1) coincides with the pointwise validity of either $d f(x)\left(T_{x} \mathbb{R}^{2}\right) \subset H_{f(x)} \mathbb{H}^{1}$ or equivalently $\operatorname{Tan}(S, f(x))=$ $H_{f(x)} \mathbb{H}^{1}$, where $S$ is parametrized by $f$.

Theorem 1. There do not exist horizontal Sobolev surfaces in $\mathbb{H}^{1}$. 
Proof. By contradiction, we assume that $\Sigma$ is a horizontal Sobolev surface in $\mathbb{H}^{1}$. Then we have an $f \in W_{l o c}^{1,1}\left(\Omega, \mathbb{H}^{1}\right)$ that is the graph of some $W_{l o c}^{1,1}$-function and such that $f(\Omega)$ is $\mathcal{H}_{|\cdot|}^{2}$-a.e. tangent to $H \mathbb{H}^{1}$. If we could find a set $E \subset \Omega$ of positive measure where (10) fails to hold, then by Theorem 1.2 of [10 and in view of Remark 4 , we would get a subset $f(E) \subset \Sigma$ of positive measure $\mathcal{H}_{|\cdot|}^{2}$ that is a.e. not tangent to $H \mathbb{H}^{1}$. This conflicts with our assumption of horizontality; hence we have proved that (10) holds a.e. in $\Omega$. The latter assertion conflicts with Lemma 1 and concludes the proof.

Remark 5. Notice that, by definition, each Sobolev surface has positive measure $\mathcal{H}_{|\cdot|}^{2}$; hence one immediately observes that it also has positive measure $\mathcal{H}^{2}$. On the other hand, $\mathcal{H}_{|\cdot|}^{2}$-negligible sets cannot have positive measure $\mathcal{H}^{3}$, since this measure is absolutely continuous with respect to $\mathcal{H}_{|\cdot|}^{2}$, as has been shown in [3].

Theorem 2. Every Sobolev surface $\Sigma \subset \mathbb{H}^{1}$ satisfies $\mathcal{H}^{3}(\Sigma)>0$.

Proof. By definition of a Sobolev surface, we can find a precisely represented function $u \in W_{\text {loc }}^{1,1}(\Omega)$, where $\Omega$ is an open subset of $\mathbb{R}^{2}$, such that the graph of $u$ is contained in $\Sigma$. Suppose that the graph is of the form

$$
\Omega \ni\left(x_{1}, x_{2}\right) \longrightarrow f\left(x_{1}, x_{2}\right)=\left(u\left(x_{1}, x_{2}\right), x_{1}, x_{2}\right) .
$$

Lemma 1 ensures that there is a subset $A \subset \Omega$ of positive measure such that

$$
\left\{\begin{array}{l}
u-x_{1} u_{x_{1}}=0 \\
1+x_{1} u_{x_{2}}=0
\end{array} \quad \text { does not hold at every point of } A .\right.
$$

Taking into account the classical Whitney extension theorem, see for instance 3.1.15 of [5] and the lemma of Section 3 in [6], one can find a bounded subset with positive measure $A_{0} \subset A$ and a $C^{1}$ smooth function $v: \mathbb{R}^{2} \longrightarrow \mathbb{R}$ such that $u$ is everywhere differentiable in $A_{0}$ and coincides there with $v$ along with its gradient. We define the submanifold

$$
\Sigma_{1}=\left\{\left(y_{1}, y_{2}, y_{3}\right) \in \mathbb{H}^{1} \mid v\left(y_{2}, y_{3}\right)-y_{1}=0,\left(y_{2}, y_{3}\right) \in \Omega\right\} .
$$

To avoid ambiguity, we have introduced the variables $\left(y_{1}, y_{2}, y_{3}\right)$, since $y_{2}$ and $y_{3}$ correspond to $x_{1}$ and $x_{2}$, respectively, of (2). Taking into account formulae (5.1) and (5.2) of [2] for $n=1$, we have

$$
\mathcal{S}^{3}\left\llcorner\Sigma_{1}=\left|\mathbf{n}_{H}\right| d \mathcal{H}_{|\cdot|}^{2}\left\llcorner\Sigma_{1},\right.\right.
$$

where $\mathcal{S}^{3}$ is the spherical Hausdorff measure with respect to a fixed Heisenberg metric. The length of the horizontal normal with respect to the Euclidean metric is given by

$$
\left|\mathbf{n}_{H}(v(y), y)\right|^{2}=\left(1+y_{2} v_{y_{3}}(y)\right)^{2}+\left(v_{y_{2}}(y)+v(y) v_{y_{3}}(y)\right)^{2}
$$

since it is equal to $\left\langle\mathbf{n}(v(y), y), X_{1}(n(v(y), y))\right\rangle^{2}+\left\langle\mathbf{n}(n(v, y)), X_{2}(n(v, y))\right\rangle^{2}$, where we have set $y=\left(y_{2}, y_{3}\right)$. Taking into account (3), for every $y \in A_{0}$, we have that $v(y)=u(y)$ and either

$$
\left|v(y)-y_{2} v_{y_{2}}(y)\right|>0 \quad \text { or } \quad\left|1+y_{2} v_{y_{3}}(y)\right|>0 .
$$

If $\left(1+y_{2} v_{y_{3}}(y)\right) \neq 0$ on a subset $E \subset A_{0}$ of positive measure, then $\left|\mathbf{n}_{H}(v(y), y)\right|>0$ for every $y \in E$. By Theorem 1.2 of [10], $f$ preserves $\mathcal{H}_{|\cdot|}^{2}$-negligible sets and also $\mathcal{H}_{|\cdot|}^{2}(f(E))>0$. As a result, due to (4) we get $\mathcal{S}^{3}(f(E))>0$, where $f(E) \subset \Sigma$. The 
remaining case is that $1+y_{2} v_{y_{3}}(y)=0$ for a.e. $y \in A_{0}$. In particular, $y_{2} \neq 0$ and $\left|v(y)-y_{2} v_{y_{2}}(y)\right|>0$ for a.e. $y \in A_{0}$. As a consequence,

$$
0<\left|y_{2} v_{y_{2}}(y)-v(y)\right|=\left|y_{2}\right|\left|v_{y_{2}}(y)+v(y) v_{y_{3}}(y)\right| \leq|y|\left|\mathbf{n}_{H}(v(y), y)\right|
$$

for a.e. $y \in A_{0}$. Thus, arguing as before, we get $\mathcal{S}^{3}\left(f\left(A_{0}\right)\right)>0$, where $f\left(A_{0}\right) \subset \Sigma$. This concludes the proof in the case that the graph has the form (2). The remaining two cases have analogous proofs.

Corollary 1. There do not exist Sobolev surfaces $\Sigma$ in $\mathbb{H}^{1}$ such that $0<\mathcal{H}^{2}(\Sigma)<$ $\infty$.

This corollary answers a question raised in [4 by Z. M. Balogh, R. HoeferIsenegger and J. T. Tyson about the possibility of constructing sets with finite and positive measure $\mathcal{H}^{2}$ with regularity between BV and Lipschitz. The authors show that there exist graphs of BV functions that have this property, although this is not true for Lipschitz parametrizations, as has been shown in 1 by L. Ambrosio and B. Kirchheim. Precisely, Lipschitz parametrizations from $\mathbb{R}^{2}$ to $\mathbb{H}^{1}$ are considered with respect to the Carnot-Carathéodory distance of $\mathbb{H}^{1}$, and this also implies the local Lipschitz property with respect to the Euclidean distance fixed in $\mathbb{H}^{1}$. Here we wish to mention that Lipschitz maps between stratified groups a.e. satisfy their associated contact equations, [9], and these equations in our case exactly correspond to the system (11).

Remark 6. Notice that the previous lemma precisely shows that the closure of the set where (11) fails to hold coincides with $\Omega$. On the other hand, it is still possible to construct even $C^{1, \alpha}$ parametrizations of graphs in $\mathbb{H}^{1}$, with $0<\alpha<1$, where (11) holds in a subset of positive measure, [2]. Then this subset must have empty interior.

It is natural to consider our previous results for parametrized surfaces, that are not necessarily graphs. In fact, one can extend the notion of a Sobolev surface to suitable images of Sobolev mappings. Clearly, this is a weaker notion than the previous one.

Definition 3. We say that a countably $\mathcal{H}_{|\cdot|}^{2}$-rectifiable set $S$ of $\mathbb{H}^{1}$ is a parametrized $W^{1, p}$-Sobolev surface if it can be written, up to $\mathcal{H}_{|\cdot|}^{2}$-negligible sets, as the countable union of images of $W_{\text {loc }}^{1, p}$-Sobolev mappings on open subsets of $\mathbb{R}^{2}$ that send $\mathcal{H}_{|\cdot|^{-}}^{2}$ negligible sets into $\mathcal{H}_{|\cdot|}^{2}$-negligible sets and that have a.e. maximal rank.

Although in the previous definition rectifiability is a consequence of the assumptions on the Sobolev parametrizations, we prefer stressing this important property.

Remark 7. In Definition 3, we have assumed also a sort of Lusin's condition on the parametrization, namely, that of preserving $\mathcal{H}_{|\cdot|}^{2}$-negligible sets. This is an important assumption, since one can find for instance Sobolev mappings of $W^{1,2}\left(\mathbb{R}^{2}, \mathbb{R}^{3}\right)$ whose image coincides with all of $\mathbb{R}^{3}$; see 7 for more general results in this vein.

Remark 8. It is also natural to assume that the Sobolev parametrizations considered in Definition 3 have a.e. maximal rank. In fact, without this assumption one can consider the smooth mapping $\left\{x \in \mathbb{R}^{2}|0<| x \mid<1\right\} \ni x \longrightarrow(0,0,|x|) \in \mathbb{H}^{1}$ whose image has positive and finite measure $\mathcal{H}^{2}$, as was already pointed out in [4. 
Definition 4 (Distributional Jacobian). Let $F \in W^{1, \frac{n^{2}}{n+1}}\left(\Omega, \mathbb{R}^{n}\right)$, where $\Omega$ is an open set of $\mathbb{R}^{n}$. Then for any $\varphi \in C_{c}^{\infty}(\Omega)$ the distributional Jacobian is defined as

$$
\left\langle\mathcal{J}_{F}, \varphi\right\rangle=-\int_{\Omega} f^{1} d \varphi \wedge d f^{2} \wedge \cdots \wedge d f^{n}
$$

where $F=\left(f^{1}, \ldots, f^{n}\right)$.

By the classical Sobolev embedding theorem, the previous definition is well posed. Using Calderón-Zygmund differentiability of Sobolev functions, S. Müller in [11] proved the validity of the following theorem; see also Section 7.1 of $[8$.

Theorem 3. Let $\Omega$ be an open subset of $\mathbb{R}^{n}$ and let $F \in W^{1, \frac{n^{2}}{n+1}}\left(\Omega, \mathbb{R}^{n}\right)$. Let $\Phi_{t}=t^{-n} \Phi\left(t^{-1} x\right)$, where $\Phi \in C_{c}^{\infty}\left(\left\{x \in \mathbb{R}^{n}:|x|<1\right\}\right)$ and $\int_{\mathbb{R}^{n}} \Phi(x) d x=1$. Then defining $\mathcal{J}_{F} * \Phi_{t}(x)=\left\langle\mathcal{J}_{F}, y \rightarrow \Phi_{t}(x-y)\right\rangle$, it follows that for a.e. $x \in \Omega$ the limit

$$
\lim _{t \rightarrow 0^{+}} \mathcal{J}_{F} * \Phi_{t}(x)=J(x, F)
$$

holds, where $J(x, F)=\operatorname{det}\left(\left(f_{x_{j}}^{i}(x)\right)_{i, j=1, \ldots, n}\right)$.

Lemma 2. Let $\Omega$ be an open subset of $\mathbb{R}^{2}$ and $f \in W_{\text {loc }}^{1,4 / 3}\left(\Omega, \mathbb{H}^{1}\right)$ be a Sobolev mapping with a.e. maximal rank. Then the conclusions of Lemma 1 still hold.

Proof. Suppose by contradiction that (1) holds a.e. in $\Omega$. Then it can be written as

$$
d f^{3}=f^{1} d f^{2}-f^{2} d f^{1}
$$

Set $F=\left(f^{1}, f^{2}\right): \Omega \longrightarrow \mathbb{R}^{2}$ and observe that in view of Definition 4 the distributional Jacobian $\mathcal{J}_{F}$ is well defined. Then the weak exterior differential of $f^{1} d f^{2}-f^{2} d f^{1}$ is vanishing. In fact, we have

$$
0=\int_{\Omega} d \varphi \wedge d f^{3}=\int_{\Omega} f^{1} d \varphi \wedge d f^{2}-\int_{\Omega} f^{2} d \varphi \wedge d f^{1}=-2\left\langle\mathcal{J}_{F}, \varphi\right\rangle .
$$

The last identities can be proved using standard smooth convolutions. In particular,

$$
\left\langle\mathcal{J}_{F}, \varphi\right\rangle=-\int_{\Omega} f^{1} d \varphi \wedge d f^{2}=0 \quad \text { for every } \varphi \in C_{c}^{\infty}(\Omega) .
$$

Due to Theorem [3, for a.e. $x \in \Omega$ there exists the following:

$$
\lim _{t \rightarrow 0^{+}} \mathcal{J}_{F} * \Phi_{t}(x)=J(x, F),
$$

where $J(x, F)=\operatorname{det}\left(\left(f_{x_{j}}^{i}\right)_{i, j=1,2}\right)$ is the pointwise Jacobian. Since the distributional Jacobian is vanishing, we have that $J(x, F)=0$ for a.e. $x \in \Omega$. Taking into account that (1) corresponds to $\nabla f^{3}=f^{1} \nabla f^{2}-f^{2} \nabla f^{1}$ a.e. in $\Omega$, we have proved that the rank of $f$ is a.e. less than or equal to one. This conflicts with our assumptions on $f$.

Remark 9. The previous lemma relies on the notion of a distributional Jacobian and its properties. We direct the reader to the recent monograph $[8$ for a thorough presentation of this topic, along with a number of related arguments.

Lemma 3. Let $f \in W_{\text {loc }}^{1,1}\left(\Omega, \mathbb{R}^{n}\right)$, where $\Omega \subset \mathbb{R}^{k}$ is an open subset and $k \leq n$ and suppose that $f$ preserves $\mathcal{H}_{|\cdot|}^{k}$-negligible sets. Then the following area formula holds:

$$
\int_{E} J f(x) d x=\int_{\mathbb{R}^{n}} N_{f}(y, E) d \mathcal{H}_{|\cdot|}^{k}(y),
$$


where $E$ is a measurable set in $\Omega$ and $J f(x)$ denotes the Jacobian of the approximate differential of $f$ at $x$.

Proof. One argues as in [6. In fact, the area formula holds for Lipschitz mappings and it is possible to find an increasing sequence of measurable sets $X_{k}$, whose union gives $E$ up to an $\mathcal{H}_{|\cdot|}^{k}$-negligible set and such that $f_{\mid X_{k}}$ is Lipschitz. By our assumption, $\mathcal{H}_{|\cdot|}^{k}\left(f\left(E \backslash \bigcup_{k} X_{k}\right)\right)=0$; hence the Beppo-Levi convergence theorem concludes the proof.

Theorem 4. There do not exist horizontal parametrized $W^{1, p}$-Sobolev surfaces in $\mathbb{H}^{1}$ for every $p \geq 4 / 3$.

Proof. Let $\Sigma$ be a parametrized $W^{1,4 / 3}$-Sobolev surface. By definition we can find a Sobolev mapping $f \in W^{1,4 / 3}\left(\Omega, \mathbb{H}^{1}\right)$ with a.e. maximal rank that sends $\mathcal{H}_{|\cdot|^{-}}^{2}$ negligible sets into $\mathcal{H}_{|\cdot|}^{2}$-negligible sets and such that $f(\Omega) \subset \Sigma$. By contradiction, suppose that $\Sigma$ is horizontal. We wish to prove that $d f(x)\left(T_{x} \mathbb{R}^{2}\right) \subset H_{f(x)} \mathbb{H}^{1}$ a.e. in $\Omega$. In fact, if this were not the case, then one could find a set of positive measure $E$ in $\Omega$ where the previous condition of horizontality does not hold. By the area formula (6) and the hypothesis on the rank of $f$ we would get $\mathcal{H}_{|\cdot|}^{2}(f(E))>0$, where $f(E)$ is not tangent to $H \mathbb{H}^{1}$ at an $\mathcal{H}_{|\cdot|^{-}}^{2}$-a.e. point. This conflicts with our hypothesis on $\Sigma$. Since $d f(x)\left(T_{x} \mathbb{R}^{2}\right) \subset H_{f(x)} \mathbb{H}^{1}$ is equivalent to the validity of (1) at $x$, we have proved the a.e. validity of (11) in $\Omega$, and this conflicts with Lemma 2 ,

Theorem 5. Let $p \geq 4 / 3$ and let $\Sigma$ be a parametrized $W^{1, p}$-Sobolev surface in $\mathbb{H}^{1}$. Then $\mathcal{H}^{3}(\Sigma)>0$.

Proof. By hypothesis, we have a $W_{\text {loc }}^{1, p}$-mapping $f: \Omega \longrightarrow \mathbb{H}^{1}$ with a.e. maximal rank that sends $\mathcal{H}_{|\cdot|}^{2}$-negligible sets into $\mathcal{H}_{|\cdot|}^{2}$-negligible sets and such that $f(\Omega) \subset \Sigma$. By Lemma 2, one can find a set $E \subset \Omega$ of positive measure such that $f$ is everywhere approximately differentiable on $E$ and the system (11) fails to hold everywhere on this set. It is also not restrictive to assume that each point of $E$ is a density point and that the approximate differential has maximal rank. Again, by the Whitney extension theorem and the lemma of Section 3 in [6], one can find a subset $E_{0}$ of $E$ with positive measure and a $C^{1}$ mapping $g: \mathbb{R}^{2} \longrightarrow \mathbb{H}^{1}$ such that $g_{\mid E_{0}}=f_{\mid E_{0}}$ and the approximate differential of $f$ along with the differential of $g$ coincide on $E_{0}$. Let us fix $x_{0} \in E_{0}$ and notice that for a fixed $r_{0}>0$ sufficiently small we have both $\mathcal{L}^{2}\left(B_{x_{0}, r_{0}} \cap E_{0}\right)>0$ and $g\left(B_{x_{0}, r_{0}}\right)=\Sigma_{0} \subset \mathbb{H}^{1}$ is an embedded surface. Up to possibly shrinking $S_{0}$, it is not restrictive to assume that it is a graph around $x_{0}$. As in the proof of Theorem 2, we apply (4), then getting

$$
\mathcal{S}^{3}\left\llcorner\Sigma_{0}=\left|\mathbf{n}_{H}\right| d \mathcal{H}_{|\cdot|}^{2}\left\llcorner\Sigma_{0} .\right.\right.
$$

By properties of $g$ and (6), we have $S_{0}=f\left(B_{x_{0}, r_{0}} \cap E_{0}\right) \subset \Sigma_{0} \cap \Sigma$ and $\mathcal{H}_{|\cdot|}^{2}\left(S_{0}\right)>0$. Since (11) does not hold on $E_{0}$, then $\mathbf{n}_{H}(f(x)) \neq 0$ for every $x \in E_{0}$; hence (7) gives $\mathcal{S}^{3}\left(S_{0}\right)>0$. This concludes the proof.

Corollary 2. For every $p \geq 4 / 3$, there do not exist parametrized $W^{1, p}$-Sobolev surfaces $\Sigma$ such that $0<\mathcal{H}^{2}(\Sigma)<\infty$.

As a final comment, we wish to point out how this note leaves open the question about existence of horizontal parametrized $W^{1, p}$-Sobolev surfaces with $1 \leq p<4 / 3$. 


\section{ACKNOWLEDGEMENT}

It is a great pleasure to thank Tadeusz Iwaniec for pleasant discussions and for his kind suggestion about Lemma 2 .

\section{REFERENCES}

[1] L. Ambrosio, B. Kirchheim, Rectifiable sets in metric and Banach spaces, Math. Ann. 318, 527-555 (2000). MR1800768 (2003a:28009)

[2] Z.M. BALOGH, Size of characteristic sets and functions with prescribed gradients, J. Reine Angew. Math. 564, 63-83 (2003). MR2021034 (2005d:43007)

[3] Z.M. Balogh, M. Rickly, F. Serra Cassano, Comparison of Hausdorff measures with respect to the Euclidean and the Heisenberg metric, Pub. Mat. 47, 237-259 (2003). MR1970902 (2004e:28007)

[4] Z.M. BAlogh, R. Hoefer-Isenegger, J.T. Tyson, Lifts of Lipschitz maps and horizontal fractals in the Heisenberg group, Ergodic Theory Dynam. Systems 26, no. 3, 621-651 (2006). MR2237461 (2007i:28009)

[5] H. FEDERER, Geometric Measure Theory, Springer (1969). MR0257325 (41:1976)

[6] P. HajŁasz, Change of variables formula under minimal assumptions, Colloq. Math. 64, 93-101 (1993). MR1201446 (94a:26027)

[7] J.T. Tyson, P. HajŁasz, Sobolev Peano cubes, Michigan Math. J. 56, 687-702 (2008). MR2490654

[8] T. Iwaniec, G. Martin, Geometric Function Theory and Non-linear Analysis, Oxford University Press (2001). MR.1859913 (2003c:30001)

[9] V. Magnani, Contact equations, Lipschitz extensions and isoperimetric inequalities, preprint (2009).

[10] J. Malý, D. Swanson, W.P. Ziemer, The co-area formula for Sobolev mappings, Trans. Amer. Math. Soc. 355, no. 2, 477-492 (2003). MR.1932709 (2004a:46037)

[11] S. Müller, Det $=\operatorname{det}$. A remark on the distributional determinant, C. R. Acad. Sci. Paris Sér. I Math. 311, no. 1, 13-17 (1990). MR1062920(92c:35042)

Dipartimento di Matematica, Università di Pisa, Largo Bruno Pontecorvo 5, 56127, Pisa, ITALY

E-mail address: magnani@dm.unipi.it 